\title{
Diffusion Kurtosis Imaging Detects Acute Microstructural Changes in White and Gray Matter after Alcohol Intoxication in Rat
}

\author{
Xi-Ran $\mathrm{CHEN}^{1}$, Jie-Ying ZENG ${ }^{1}$, Ling-Mei KONG ${ }^{1}$, Wen-Bin \\ ZHENG ${ }^{1, *}$
}

${ }^{1}$ Departments of Radiology, the Second Affiliated Hospital, Medical College of Shantou University, Shantou, China

Email:316980079@qq.com

${ }^{*}$ Corresponding author

Keywords: Magnetic resonance imaging, Diffusion kurtosis imaging, Alcohol Intoxication, Rat brain.

\begin{abstract}
Diffusion kurtosis imaging (DKI) is an extension of Diffusion tensor imaging (DTI) but based on a non-gaussian diffusion model, allowing more comprehensive characterization of microstructural changes in the brain. The aim of this research was to test the technical feasibility to microstructural changes in the brain of rats with acute alcohol intoxication. Changes in DTI parameters and DKI parameters in the cerebral cortex and the thalamus were investigated in the acute alcohol intoxication rat models $(\mathrm{n}=25)$ at the acute $(30 \mathrm{~min})$ stages following ethyl alcohol $(\mathrm{EtOH})$ administration. Fractional anisotropy (FA), mean diffusivity (MD) and mean kurtosis $(\mathrm{MK})$ both show significant decrease in the cortex (FA: $\mathrm{p}=0.011, \mathrm{MD}: \mathrm{p}<0.01$, MK: $\mathrm{p}<0.01$ ). However, reduced MK and MD ( $\mathrm{p}<0.01$, all) observed in the thalamus while no change here observed with FA. HE staining revealed neurons in edema and disorder, proliferation but loss arrangement of nerve fibers and with some neurons displayed the changes of necrosis and apoptosis. These data demonstrate that DKI may be sensitive in tracking pathophysiological changes associated with acute alcohol intoxication and may provide additional information which may be missed by conventional DTI parameters along.
\end{abstract}

\section{Introduction}

Alcohol abuse represents a substantial public health problem worldwide. Acute alcohol intoxication, which is one of the main alcohol-related damages, is the major factor contributing to premature death. Although EtOH ingestion impacts most organ systems, its effects on the brain are of vital importance, given EtOH's many neurological changes, such as the blood flow, electrical brain activities, memory function[1,2].

Understanding tissue alterations at an early stage following alcohol intoxication is critical for management and prevention of more severe secondary damage to the brain. However, both CT and conventional MR imaging usually fail to detect any macroscopic and microscopic evidence of brain injury in acute alcoholism[3].

Recent advances in neuroimaging techniques hold promise to be more sensitive and accurate in the detection and characterization of microstructural changes that occur in the brain due to acute alcoholism[4]. In recent years, DTI has been found to be sensitive to EtOH-induced changes in the brain especially in regional white matter(WM), which can detect microscopic tissue damage in acute alcohol intoxication $[5,6]$. The theory on which DTI is based assumes that water molecules move through a homogeneous 
environment in which diffusion can be estimated using a Gaussian displacement probability distribution[7].

Most biological tissues, especially brain tissues, however, have an intricate microstructure that restricts the movement of water, and causes diffusion to deviate substantially from a Gaussian form. This means that DTI is a limited indicator of complexity. Therefore, more dedicated methods for diffusion have been introduced [8]. The most popular method proposed is the DKI method, which is a recently developed noninvasive MRI technique that measures non-gaussian properties of water diffusion [9, 10], has been shown to be effective for monitoring microstructural changes especially in grey matter(GM), such as cerebral ischemia [11, 12], traumatic brain injury $[13,14]$, neuropsychiatric disease $[15]$ and changes in the brain with aging and degenerative disease [16, 17].

Given the sensitivity of DKI to changes in tissue microstructure we hypothesize that it may play an important role in detecting brain tissue changes following acute alcoholism. To test this hypothesis, we investigated DKI parameters on rats with acute alcoholism at the acute stage and compared the findings with the tissue histopathology.

\section{Material and Methods}

\section{Animal Models}

All animal experiments were conducted at the Department of Medical Imaging of the Second Affiliated Hospital after approval by the ethics committee of Shantou Medical University. Adult male Sprague-Dawley rats $(n=25,250-350$ grams, from the Laboratory Animal Center of Shantou Medical University, CHINA) were used in the study, which were divided randomly into two groups: group I (control, $n=5$ ) and group II (rats with acute alcoholic intoxication, $\mathrm{n}=20$ ). For EtOH administration, rats received intraperitoneal injection of EtOH (about $1.2 \mathrm{ml} / 100 \mathrm{~g}$, Hongxing Erguotou wine, $56 \%$ vol, Beijing, China), then rats were loss of righting reflex (LORR), proved successful model [18]. Animals of the control group in our study were given $1.2 \mathrm{ml} / 100 \mathrm{~g}$ of saline by intraperitoneal injection. After the experiment, all the rats were killed without suffering.

\section{Imaging}

All experiments were performed on the Varian machine including a 7.0 T horizontal magnet equipped with a Varian INOVA console. Anatomic MRI and in vivo DKI were acquired with an elliptical surface RF coil with long axis $2 \mathrm{~cm}$ and short axis $1.2 \mathrm{~cm}$. The measurements of DKI were collected $30 \mathrm{~min}$ after the onset of LORR in rats. At all times during the experiment, the animal was under $1-2 \%$ isoflurane anesthesia and 1 $\mathrm{L} / \mathrm{min}$ oxygen administration.

A three-slice (axial, mid-sagittal, and coronal) scout using rapid acquisition with fast low angle shot (FLASH) was used to localize the rat brain. T2-weighted imaging was obtained using a 2D rapid acquisition with Fast spin-echo sequence with a 2000ms repetition time (TR), a $10 \mathrm{~ms}$ echo time, mean equal to 4 , a $3 \mathrm{~cm}$ field of view(FOV), and an in-plane resolution of $180 \mu \mathrm{m}$ using 5 slices at $2 \mathrm{~mm}$ thickness with 0.2 gap. The T2-weighted imaging was used to position the DKI voxel.

For the DKI acquisition, it acquired with fast spin echo multislice (FSEMS) sequence, together with a TR/TE of $2000 / 36 \mathrm{~ms}$ at $2 \mathrm{~mm}$ thickness with 0.2 gap, 2 averages, 4 shots and 16 kzero. Two b-values (1000 s/mm2 and $2000 \mathrm{~s} / \mathrm{mm} 2)$ were 
acquired for 30 direction following the acquisition of image acquired at $b=0 \mathrm{~s} / \mathrm{mm} 2$. The average duration time for the DKI scan was approximately $33 \mathrm{~min}$.

Shimming was often challenging likely because of the rat breathing. If repeated shimming attempts could not achieve a line width of less than $20 \mathrm{~Hz}$, then pictures were not collected.

\section{Histology}

After all images were acquired, animals were immediately sacrificed for histology. Animals received an overdose of chloral hydrate and were perfused transcardially with 4\% paraformaldehyde in phosphate buffer. Brains were removed and fixed with $4 \%$ paraformaldehyde for $48 \mathrm{~h}$. After fixation, brains were embedded in paraffin, and contiguous $5 \mu \mathrm{m}$ sections at the level of the brain were cut on a microtome $(\mathrm{Rm} 2016$, LEICA, Germany). Sections were stained with Hematoxylin and Eosin (HE) staining.

\section{Data Processing}

The DKI data were processed with MATLAB (The Mathworks, Natick, MA) scripts. Three-dimensional motion correction and spatial smoothing (gaussian filter full-widthhalf-maximum; FWHM $=2.5 \mathrm{~mm}$ ) were performed with the Statistical Parametric Mapping package (SPM; Wellcome Trust Centre for Neuroimaging at University College London, London, U.K.).

Analyses of the MK, AK, RK, FA and MD maps were performed with MRIcro (Neuropsychology Lab, Columbia SC, USA) software. Anatomical regions of interest (ROIs) were selected on T2-weighted scans by two experienced observer, and then transferred onto co-registered MK, AK, RK, FA and MD maps. Rectangular ROIs were placed bilaterally on two spacing distance in the thalamus and cerebral cortex. The average MK, AK, RK, FA and MD were calculated for each ROI, and these values were then averaged over all spaces and both sides to derive one mean for specified brain region on every map.

\section{Statistical Analysis}

Each of the measured parameters (MD, FA, MK, AK and RK) was expressed as mean \pm standard deviation. Data analysis was carried out using SPSS 17.0 (SPSS Inc., Chicago, Illinois, USA) with an independent-samples t-test to compare groups. All reported $p$ values were corrected and statistical significance was deemed at $p<0.05$.

\section{Results}

All the animals survived during the whole scan time. Parametric maps of DTI (MD, FA) and DKI (MK, AK, RK) were generated for each of the animals. Fig.1 shows MK, FA and MD maps from a representative rat on the control group and acute alcoholism group. Comparisons of regional DTI and DKI measures between controls and rats with acute alcoholism in different ROI are reported in Table.1. A significant difference was detected in $\mathrm{MK}$ and $\mathrm{MD}$, compared to controls, the group of acute alcoholism showed significantly lower MK and MD in the cerebral cortex $(\mathrm{p}<0.01)$ and the thalamus $(\mathrm{p}<0.01)$. Meanwhile, AK was decreased in the cerebral cortex $(\mathrm{p}=0.012)$ and increased in the thalamus $(\mathrm{p}=0.163)$. $\mathrm{RK}$ was reduced in both the cerebral cortex $(\mathrm{p}=0.032)$ and the thalamus $(\mathrm{p}<0.01)$. Lower FA was seen in the cerebral cortex $(\mathrm{p}=0.011)$, and no differences detected in the thalamus $(\mathrm{p}=0.110)$.

Fig.2 shows histology using HE staining from representative rat at the acute stage post $\mathrm{EtOH}$ administration compared to a sham rat. In the control group, HE staining 
revealed normal pericapillary spaces and intact vessel walls within the cerebral cortex and thalamus. Meanwhile, no erythrocytes were observed within the vascular lumina. Neurons exhibited normal morphology, with clear round nuclei and a constant nucleus-cytoplasm ratio. For rats with acute alcoholism, HE staining mainly revealed neurons in edema and disorder, proliferation but loss arrangement of nerve fibers and with some neurons displayed the changes of necrosis and apoptosis.

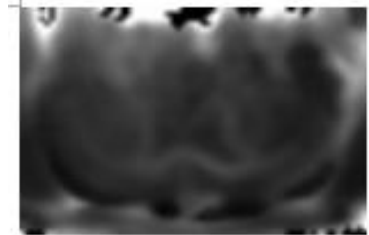

a

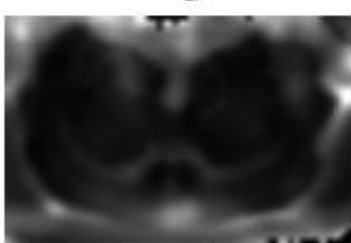

d

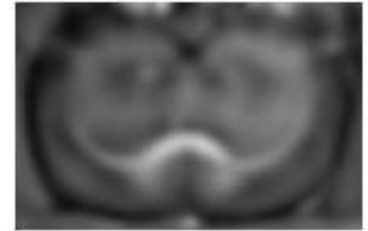

b

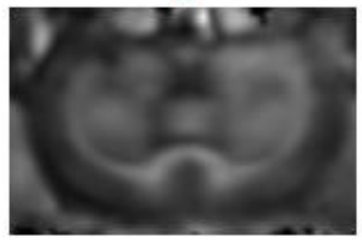

e

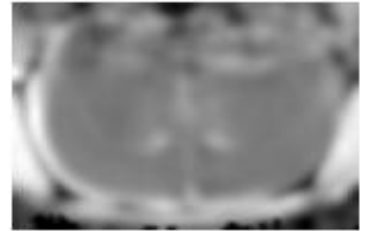

C

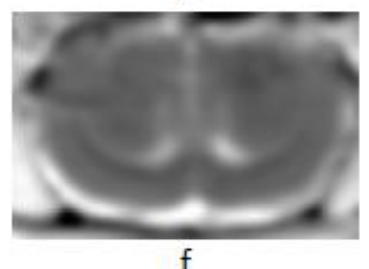

Figure 1. MK, FA and MD maps of control and $0.5 \mathrm{~h}$ after EtOH aministration. There is hypointensitity on the MK, FA and MD imaging in cerebral cortex and hypointensitity on the MK and MD imaging in thalamus.(a-c: MK, FA and MD values in the control group; d-e: MK, FA and MD values in the experiment group)
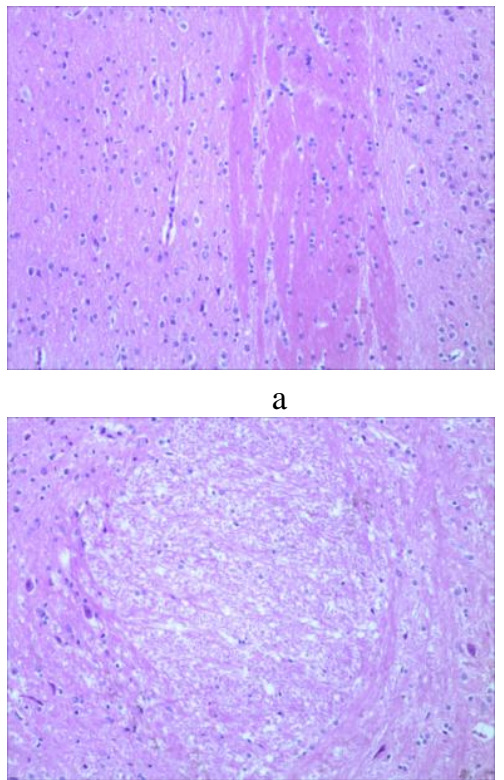

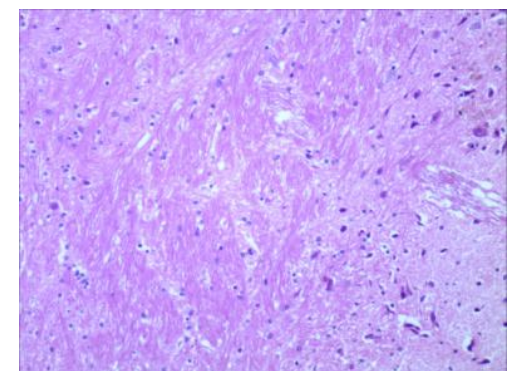

b

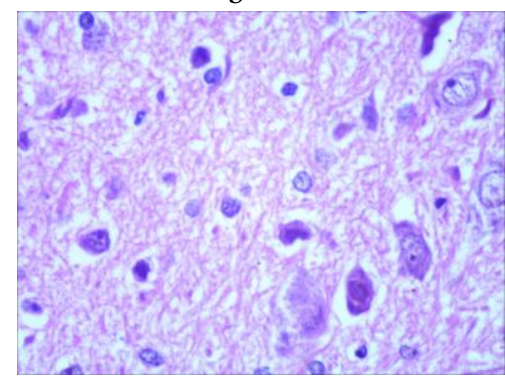

d

Figure 2. Comparison of HE stains in representative acute alcoholism rat and a sham rat. Acute alcoholism rat mainly expressed the cell edema and disorder, proliferation but loss arrangement of nerve fibers and some neurons necrosis and apoptosis.(a: control group; b-d: acute alcoholism group) 
Table 1. Comparisons of regional DKI and DTI measures between controls and rats with acute alcoholism in cerebral cortex and thalamus (mean \pm SD)

\begin{tabular}{|c|c|c|c|c|c|c|c|c|c|c|}
\hline \multirow{2}{*}{ groups } & \multicolumn{5}{|c|}{ cerebral cortex } & \multicolumn{5}{|c|}{ thalamus } \\
\hline & $\mathrm{MK}$ & $\mathrm{AK}$ & $\mathrm{RK}$ & FA & MD & $\mathrm{MK}$ & $\mathrm{AK}$ & $\mathrm{RK}$ & FA & MD \\
\hline \multirow{2}{*}{ control } & $0.672 \pm$ & $0.753 \pm$ & $0.625 \pm$ & $0.257 \pm$ & $0.800 \pm$ & $0.690 \pm$ & $0.518 \pm$ & $0.910 \pm$ & $0.397 \pm$ & $0.780 \pm$ \\
\hline & 0.181 & 0.211 & 0.232 & 0.129 & 0.086 & 0.181 & 0.227 & 0.188 & 0.123 & 0.048 \\
\hline \multirow{2}{*}{ alcoholism } & $0.375 \pm$ & $0.504 \pm$ & $0.404 \pm$ & $0.192 \pm$ & $0.650 \pm$ & $0.425 \pm$ & $0.651 \pm$ & $0.564 \pm$ & $0.345 \pm$ & $0.623 \pm$ \\
\hline & $0.137^{* *}$ & $0.172^{*}$ & $0.157^{*}$ & $0.042^{* *}$ & $0.046^{* *}$ & $0.191^{* *}$ & 0.239 & $0.202^{* *}$ & 0.072 & $0.088^{* * *}$ \\
\hline
\end{tabular}

$-\mathrm{P}<0.01$ was considered to indicate an obviously statistically sionificant difference.

\section{Discussion}

This study shows that DKI measures provide higher detection and better characterization of regional brain injury including WM and GM in acute alcoholism rat models. More specifically, our study indicates that DKI have the potential to advance our understanding of pathophysiology in acute alcoholism, and may possibly yield valuable predictors for the severity of brain damage and cognitive outcome.

FA, a normalized measure of anisotropy calculated from DTI, has been shown to be sensitive to microstructural changes in WM integrity [19]. In our study, a significant reduction in FA throughout cerebral cortex reflects the decrease in anisotropy such as axonal break-down and demyelination progresses in the WM tracts of acute alcoholism [20]. This phenomenon has also been well-documented in other acute damaging disease such as the acute stage of traumatic brain injury [13, 21]. However, low FA did not reach statistical significance in the thalamus, which is also indicated that DTI metrics only sensitive to microstructural changes in WM integrity.

MD should be interpreted in terms of changes in the diffusion coefficient of the intracellular volume relative to the intracellular volume [22]. Reduced MD suggests cytotoxic edema, whereas increased MD in the brain indicates vasogenic edema $[5,22]$. Therefore, the MD decrease seen in abnormal brain tissue can be ascribed to cellular swelling, which proved by HE staining results.

MK, as the most important index of DKI, defined as the average of the kurtosis over all possible diffusion direction $[8,23]$. In general, the more complex the structure of ROIs, the more significant restricts the movement of water, which lead to an increase in MK. Our result reveals lower MK in the cerebral cortex and thalamus of the rats with acute alcoholism, which can be interpreted as a reduction in overall diffusional heterogeneity. This may be largely due to two reasons which have already interpretation in previous studies. On the one hand, a decrease in MK may associated with degenerative processes leading to neuronal shrinkage $[9,10,24,25]$. On the other hand, it may induced by changes in axonal and myelin density [26]. For rats with acute alcoholism, HE staining mainly showed that the arrangement of neuros and nerve fibers was disordered and some neurons necrosis and apoptosis, which is in agreement with the above research results to some extent.

The most important finding in this study is that a steady reduce in MK and no appreciable change in FA in the thalamus, which is not only one of the GM but always associated with cognitive impairment [27]. This supports the hypothesis that DKI is sensitivity to EtOH-related microstructural changes that occur in the isotropic GM and better understanding the brain cognitive outcome, for which DTI has previously been shown to have limited sensitivity.

There are several potential limitations to this study. One concern is that only HE staining was performed to obtain pathophysiology information on the animals. While 
future studies should focus on some immunohistochemistry, which may provide more accurate and better understanding of the physiological processes inducing by acute alcoholism that may have an effect on diffusion kurtosis. Nevertheless, we only choose cerebral cortex and thalamus as the ROI, it would be of great interest to see other regional brain changes through DKI metrics. Another concern is the sample size used in this study was too small and we only followed the animals for acute stage (30 min), it would be of interest to increase the sample size and trace animals to subacute even chronic phase. Last, the duration time for the DKI scan was too long which may limit its application, optimizing the scanning parameters is one of our future targets.

\section{Conclusions}

This study demonstrates that diffusion kurtosis parameters can provide additional information and observe the EtOH-related brain changes in acute stage. Similar to DTI, DKI can also detect anisotropic WM changes due to acute alcoholism. In addition, DKI can provide GM changes which may be under-estimated by parameters from DTI. In summary, DKI offers more sensitive and comprehensive measures for the quantitative evaluation of EtOH-related microstructural changes in both WM and GM and thereby may be a valuable technique for studying the pathophysiological changes associated with the sequelae following acute alcoholism.

\section{Acknowledgement}

This study was supported by the National Natural Science Foundation of China (grant No.81571627), Natural Science Foundation of Guangdong Province, China (grant No. 2014A030313481), Shantou University Innovation project, Shantou, China, and Medical Scientific Research Foundation of Guangdong Province, China (grant No.B2014284).

\section{References}

[1] Sun, C., L. Shen, X. Li, C. Liu and Y. Zhou, Risk of pneumonia in central nervous system injury with alcohol intake: a meta-analysis. Int J Clin Exp Med, 2015. 8(9): p. 15738-15744.

[2] Paul, S.M., Alcohol-sensitive GABA receptors and alcohol antagonists. Proc Natl Acad Sci U S A, 2006. 103(22): p. 8307-8.

[3] Rooney, W.D., J.H. Lee, X. Li, G.J. Wang, D. Franceschi, C.S. Springer, Jr. and N.D. Volkow, $4.0 T$ water proton $T 1$ relaxation times in normal human brain and during acute ethanol intoxication. Alcohol Clin Exp Res, 2000. 24(6): p. 830-6.

[4] Bjork, J.M. and J.M. Gilman, The effects of acute alcohol administration on the human brain: insights from neuroimaging. Neuropharmacology, 2014. 84: p. 101-10.

[5] Kong, L.M., W.B. Zheng, G.P. Lian and H.D. Zhang, Acute effects of alcohol on the human brain: diffusion tensor imaging study. AJNR Am J Neuroradiol, 2012. 33(5): p. 928-34.

[6] Liu, H., W. Zheng, G. Yan, B. Liu, L. Kong, Y. Ding, Z. Shen, H. Tan and G. Zhang, Acute ethanol-induced changes in edema and metabolite concentrations in rat brain. Biomed Res Int, 2014. 2014: p. 351903. 
[7] Hui, E.S., M.M. Cheung, K.C. Chan and E.X. Wu, B-value dependence of DTI quantitation and sensitivity in detecting neural tissue changes. Neuroimage, 2010. 49(3): p. 2366-74.

[8] Hori, M., I. Fukunaga, Y. Masutani, T. Taoka, K. Kamagata, Y. Suzuki and S. Aoki, Visualizing non-Gaussian diffusion: clinical application of $q$-space imaging and diffusional kurtosis imaging of the brain and spine. Magn Reson Med Sci, 2012. 11(4): p. 221-33.

[9] Jensen, J.H. and J.A. Helpern, MRI quantification of non-Gaussian water diffusion by kurtosis analysis. NMR Biomed, 2010. 23(7): p. 698-710.

[10] Jensen, J.H., J.A. Helpern, A. Ramani, H. Lu and K. Kaczynski, Diffusional kurtosis imaging: the quantification of non-gaussian water diffusion by means of magnetic resonance imaging. Magn Reson Med, 2005. 53(6): p. 1432-40.

[11] Hui, E.S., E. Fieremans, J.H. Jensen, A. Tabesh, W. Feng, L. Bonilha, M.V. Spampinato, R. Adams and J.A. Helpern, Stroke assessment with diffusional kurtosis imaging. Stroke, 2012. 43(11): p. 2968-73.

[12] Cheung, J.S., E. Wang, E.H. Lo and P.Z. Sun, Stratification of heterogeneous diffusion MRI ischemic lesion with kurtosis imaging: evaluation of mean diffusion and kurtosis MRI mismatch in an animal model of transient focal ischemia. Stroke, 2012. 43(8): p. 2252-4.

[13] Zhuo, J., S. Xu, J.L. Proctor, R.J. Mullins, J.Z. Simon, G. Fiskum and R.P. Gullapalli, Diffusion kurtosis as an in vivo imaging marker for reactive astrogliosis in traumatic brain injury. Neuroimage, 2012. 59(1): p. 467-77.

[14] Grossman, E.J., J.H. Jensen, J.S. Babb, Q. Chen, A. Tabesh, E. Fieremans, D. Xia, M. Inglese and R.I. Grossman, Cognitive impairment in mild traumatic brain injury: a longitudinal diffusional kurtosis and perfusion imaging study. AJNR Am J Neuroradiol, 2013. 34(5): p. 951-7.

[15] Helpern, J.A., V. Adisetiyo, M.F. Falangola, C. Hu, A. Di Martino, K. Williams, F.X. Castellanos and J.H. Jensen, Preliminary evidence of altered gray and white matter microstructural development in the frontal lobe of adolescents with attention-deficit hyperactivity disorder: a diffusional kurtosis imaging study. J Magn Reson Imaging, 2011. 33(1): p. 17-23.

[16] Blockx, I., G. De Groof, M. Verhoye, J. Van Audekerke, K. Raber, D. Poot, J. Sijbers, A.P. Osmand, S. Von Horsten and A. Van der Linden, Microstructural changes observed with DKI in a transgenic Huntington rat model: evidence for abnormal neurodevelopment. Neuroimage, 2012. 59(2): p. 957-67.

[17] Wang, J.J., W.Y. Lin, C.S. Lu, Y.H. Weng, S.H. Ng, C.H. Wang, H.L. Liu, R.H. Hsieh, Y.L. Wan and Y.Y. Wai, Parkinson disease: diagnostic utility of diffusion kurtosis imaging. Radiology, 2011. 261(1): p. 210-7.

[18] Khisti, R.T., M.J. VanDoren, T. O'Buckley and A.L. Morrow, Neuroactive steroid 3 alpha-hydroxy-5 alpha-pregnan-20-one modulates ethanol-induced loss of righting reflex in rats. Brain Res, 2003. 980(2): p. 255-65.

[19] Mukherjee, P. and R.C. McKinstry, Diffusion tensor imaging and tractography of human brain development. Neuroimaging Clin N Am, 2006. 16(1): p. 19-43, vii. 
[20] Basser, P.J., Inferring microstructural features and the physiological state of tissues from diffusion-weighted images. NMR Biomed, 1995. 8(7-8): p. 333-44.

[21] Mayer, A.R., J. Ling, M.V. Mannell, C. Gasparovic, J.P. Phillips, D. Doezema, R. Reichard and R.A. Yeo, A prospective diffusion tensor imaging study in mild traumatic brain injury. Neurology, 2010. 74(8): p. 643-50.

[22] Cernak, I., R. Vink, D.N. Zapple, M.I. Cruz, F. Ahmed, T. Chang, S.T. Fricke and A.I. Faden, The pathobiology of moderate diffuse traumatic brain injury as identified using a new experimental model of injury in rats. Neurobiol Dis, 2004. 17(1): p. 29-43.

[23] Jensen, J.H., M.F. Falangola, C. Hu, A. Tabesh, O. Rapalino, C. Lo and J.A. Helpern, Preliminary observations of increased diffusional kurtosis in human brain following recent cerebral infarction. NMR Biomed, 2011. 24(5): p. 452-7.

[24] Lu, H., J.H. Jensen, A. Ramani and J.A. Helpern, Three-dimensional characterization of non-gaussian water diffusion in humans using diffusion kurtosis imaging. NMR Biomed, 2006. 19(2): p. 236-47.

[25] Wu, E.X. and M.M. Cheung, MR diffusion kurtosis imaging for neural tissue characterization. NMR Biomed, 2010. 23(7): p. 836-48.

[26] Fieremans, E., J.H. Jensen and J.A. Helpern, White matter characterization with diffusional kurtosis imaging. Neuroimage, 2011. 58(1): p. 177-88.

[27] Ide, S., S. Kakeda and Y. Korogi, [Anatomy of the Thalamus]. Brain Nerve, 2015. 67(12): p. 1459-69. 